\title{
Numerical Experiments on the Solution of the Inverse Additive Singular Value Problem
}

\author{
G. Flores-Becerra ${ }^{1,2}$, Victor M. Garcia ${ }^{1}$, and Antonio M. Vidal ${ }^{1}$ \\ 1 Departamento de Sistemas Informáticos y Computación, \\ Universidad Politécnica de Valencia, \\ Camino de Vera s/n, 46022 Valencia, España, \\ \{gflores, vmgarcia, avidal\}@dsic.upv.es \\ 2 Departamento de Sistemas y Computación, \\ Instituto Tecnológico de Puebla, \\ Av. Tecnológico 420, Col. Maravillas, C.P. 72220, Puebla, México
}

\begin{abstract}
The work presented here is an experimental study of four iterative algorithms for solving the Inverse Additive Singular Value Problem (IASVP). The algorithms are analyzed and evaluated with respect to different points of view: memory requirements, convergence, accuracy and execution time, in order to observe their behaviour with different problem sizes and to identify those capable to solve the problem efficiently.
\end{abstract}

\section{Introduction}

Inverse problems are of interest for different applications in Science and Engineering, such as Geophysics, Computerized Tomography, Simulation of Mechanical Systems, and many more 44 9, 11, 12. Two specific inverse problems are the Inverse Eigenvalue Problem (IEP) and the Inverse Singular Value Problem (ISVP). The goal of these problems is to build a matrix with some structure features, and with eigenvalues (or singular values) previously fixed. In this paper we study a particular case of the ISVP, the Inverse Additive Singular Value Problem (IASVP), which can be defined as:

Given a set of matrices $A_{0}, A_{1}, \ldots, A_{n} \in \Re^{m \times n}(m \geq n)$ and a set of real numbers $S^{*}=\left\{S_{1}^{*}, S_{2}^{*}, \ldots, S_{n}^{*}\right\}$, where $S_{1}^{*}>S_{2}^{*}>\ldots>S_{n}^{*}$, find a vector $c=$ $\left[c_{1}, c_{2}, \ldots, c_{n}\right]^{t} \in \Re^{n}$, such that $S^{*}$ are the singular values of

$$
A(c)=A_{0}+c_{1} A_{1}+\ldots+c_{n} A_{n} .
$$

There are several well known methods for the IEP. Friedland et al. 8 pose several methods for the IEP based in Newton's method, named Method I, Method II, Method III and Method IV. Chen and Chu [2] proposed the Lift\&Project method, where the IEP is solved as a series of minimum squares problems.

The methods for the IASVP are usually derived from those for the IEP. In [3], Chu proposes two methods for the IASVP, one of them (The "discrete" 
method) is obtained by adapting the Method III for the IEP to the IASVP; we shall name this method as MIII. The convergence of MIII was proved in [5].

The MI [7] and LP [6] methods for the IASVP are other methods derived of Method I and Lift\&Project, respectively. On the other hand, the simplest way to set up a Newton iteration for resolution of the IASVP is to write directly the problem as a system of nonlinear equations; we call this a Brute Force (BF) approach.

Some experimental results of IASVP resolution were given for size of problem smaller than 6 (by example, $m=5$ and $n=4$ in 3 ] and [5]); so, it is necesary to study the behaviour of the algorithms for greater sizes.

The goal of this work has been to make a fair comparison among some of these methods with larger problem sizes than those used in previous studies. To do so, we have implemented FB, MI, MIII and LP methods. The performances of these algorithms have been analyzed and evaluated, for different values of $m$ and $n$, regarding memory requirements, convergence, solution accuracy and execution time, through an experimental study, to determine those of better characteristics to solve the IASVP.

In section 2 the algorithms are briefly described; the performance of the algorithms is analyzed in section [3, and, finally, the conclusions are given in section 4.

\section{Resolution Methods for IASVP}

\subsection{Brute Force (BF)}

Let $c^{*}$ be a solution of the IASVP; then, the singular value decomposition of $A\left(c^{*}\right)$, must be

$$
A\left(c^{*}\right)=\operatorname{Pdiag}\left(S^{*}\right) Q^{t}
$$

with $P \in \Re^{m \times n}$ and $Q \in \Re^{n \times n}$, orthogonals.

A system of nonlinear equations of the form $F(z)=0$, can be built by using (2) and the orthogonality of $P$ and $Q$. In this system, the unknown vector would be $z=\left[Q_{1,1}, Q_{1,2}, \ldots, Q_{n, n}, P_{1,1}, P_{1,2}, \ldots, P_{m, n}, c_{1}, c_{2}, \ldots, c_{n},\right]^{t}, n$ unknowns of $c$, $m n$ unknowns of $P 1$ and $n^{2}$ unknowns of $Q$, then $F(z)=0$ is $F(Q, P, c)=0$, where

$$
\begin{gathered}
F_{(i-1) n+j}(Q, P, c)=\left(A_{0}+c_{1} A_{1}+\cdots+c_{n} A_{n}-P S^{*} Q^{t}\right)_{i, j} ; i=1: m ; j=1: n ; \\
F_{m n+(i-1) n+j-i+1}(Q, P, c)=\left(P^{t} P-I_{m}\right)_{i, j} ; i=1: n ; j=i: n ; \\
F_{m n+n \frac{n+1}{2}+(i-1) n+j-i+1}(Q, P, c)=\left(Q^{t} Q-I_{n}\right)_{i, j} ; i=1: n ; j=i: n ;
\end{gathered}
$$

A nonlinear system with $m n+n^{2}+n$ equations and $m n+n^{2}+n$ unknowns has been defined from (3), (44) and (5); its solution can be approximated through Newton's method, computing a succession of $\left(Q^{(0)}, P^{(0)}, c^{(0)}\right),\left(Q^{(1)}, P^{(1)}, c^{(1)}\right)$,

\footnotetext{
${ }^{1}$ Only $m n$ unknowns of $P$ because we need only $\min \{m, n\}$ singular values.
} 
$\ldots,\left(Q^{(k)}, P^{(k)}, c^{(k)}\right)$ that approximates to the solution of $F(Q, P, c)=0$. Then, if $z^{(k)}$ is the $k$-th element of this succession, the $(k+1)$-th element is given by the expression [10]:

$$
z^{(k+1)}=z^{(k)}-J\left(z^{(k)}\right)^{-1} F\left(z^{(k)}\right)
$$

where $J\left(z^{(k)}\right)$ is the Jacobian matrix of $F(z)$ evaluated at $z^{(k)}$. The Jacobian matrix of $F(Q, P, c)$ is

$$
J(Q, P, c)=\left[\frac{\partial F_{r}(z)}{\partial z_{t}}\right]_{r=1: m n+n^{2}+n ; t=1: m n+n^{2}+n}=\left[\begin{array}{ccc}
J_{1,1} & 0 & 0 \\
0 & J_{2,2} & 0 \\
J_{3,1} & J_{3,2} & J_{3,3}
\end{array}\right]
$$

where $J_{1,1}, J_{2,2}, J_{3,1}, J_{3,2}$ y $J_{3,3}$ are blocks of size $n \frac{n+1}{2} \times n^{2}, n \frac{n+1}{2} \times m n$, $m n \times n^{2}, m n \times m n$ and $m n \times n$, respectively, such that

for $i=0: n-1 ; j=1: n ;$ row $=1+\sum_{k=0}^{i-1}(n-k) ; c o l=i+(j-1) n+1$

$$
\begin{array}{ll}
\left(J_{1,1}\right)_{\text {row }, \text { col }} & =2 Q_{j, i+1} \\
\left(J_{1,1}\right)_{\text {row }+1: \text { row }+n-i-1, \text { col }} & =Q_{j, i+2: n}^{t} \\
\left(J_{1,1}\right)_{\text {row }+a, \text { col }+a} & =Q_{j, i+1} \quad a=1: n-i-1 ;
\end{array}
$$

for $i=0: n-1 ; j=1: m ;$ row $=1+\sum_{k=0}^{i-1}(n-k) ;$ col $=i+(j-1) n+1$

$$
\begin{array}{ll}
\left(J_{2,2}\right)_{\text {row }, \text { col }} & =2 P_{j, i+1} \\
\left(J_{2,2}\right)_{\text {row }+1: \text { row }+n-i-1, \text { col }} & =P_{j, i+2: n}^{t} \\
\left(J_{2,2}\right)_{\text {row }+a, \text { col }+a} & =P_{j, i+1} \quad a=1: n-i-1 ;
\end{array}
$$

and for $i=1: m ; j=1: n ; t=1: n$

$$
\begin{aligned}
& \left(J_{3,1}\right)_{(i-1) n+j,(j-1) n+t}=S_{t}^{*} P_{i, t} \\
& \left(J_{3,2}\right)_{(i-1) n+j,(i-1) n+t}=S_{t}^{*} Q_{j, t} \\
& \left(J_{3,3}\right)_{(i-1) n+j, t}=\left(A_{t}\right)_{i, j}
\end{aligned}
$$

This iterative method converges quadratically to the solution of $F(Q, P, c)$ if the initial guess $Q^{(0)}, P^{(0)}$ and $c^{(0)}$ is close enough to the solution [10].

\section{$2.2 \quad$ MI}

As mentioned before, the MI method for the IASVP follows the ideas from Method I for IEP [8]. First, for any $c$ we can obtain the singular value decomposition of (11) as $A(c)=P(c) S(c) Q(c)^{t}$. Then, the IASVP can be stated as 
finding the solution of the nonlinear system in $c: F(c)=\left[S_{i}(c)-S_{i}^{*}\right]_{i=1, n}=0$. If Newton's method is applied to solve this nonlinear system, the Jacobian matrix is needed; it can be obtained as in [7]: $J=\left[p_{i}^{t} A_{j} q_{i}\right]_{i, j=1, n}$, and the Newton's iteration (6) is given by the expression [7]

$$
J^{(k)} c^{(k+1)}=b^{(k)}
$$

where $b^{(k)}=S^{*}-\left[p_{i}^{(k) t} A_{0} q_{i}^{(k)}\right]_{i=1, n}$. MI converges quadratically to $c^{*}$ if $c^{(0)}$ is close enough to $c^{*}[10$.

\section{$2.3 \quad$ LP}

LP is developed in a similar way to Lift\&Project in [2]. Let us define $\Gamma\left(S^{*}\right)$, the set of matrices in $\Re^{m \times n},(m \geq n)$, which can be written in the form $P S^{*} Q^{t}$, where $P \in \Re^{m \times n}$ and $Q \in \Re^{n \times n}$ are orthogonal matrices; and let $\Lambda(c)$ be the set of matrices that can be expressed as in (1). The goal is to find the intersection of both sets, using distance minimization techniques. The distance between two matrices $U$ and $V$ is defined as $d(U, V)=\|U-V\|_{F}$.

LP is an iterative algorithm, with two stages for each iteration:

1) The Lift stage, which consists in, given $c^{(k)}$ (given $A\left(c^{(k)}\right) \in \Lambda(c)$ ) find $X^{(k)} \in \Gamma\left(S^{*}\right)$ such that $d\left(A\left(c^{(k)}\right), X^{(k)}\right)=d\left(A\left(c^{(k)}\right), \Gamma\left(S^{*}\right)\right)$. This is achieved by computing the singular value decomposition of $A\left(c^{(k)}\right), P^{(k)} S^{(k)} Q^{(k) t}$, and then computing $X^{(k)}=P^{(k)} S^{*} Q^{(k) t}$, which turns out to be the element of $\Gamma\left(S^{*}\right)$ closest to $A\left(c^{(k)}\right)$ 7.

2) The Projection stage consist in, given $X^{(k)} \in \Gamma\left(S^{*}\right)$, find $c^{(k+1)}$ (find $\left.A\left(c^{(k+1)}\right) \in \Lambda(c)\right)$ such that $d\left(X^{(k)}, A\left(c^{(k+1)}\right)\right)=d\left(X^{(k)}, \Lambda\left(c^{(k+1)}\right)\right)$. This is achieved by finding $c^{(k+1)}$ as the solution of the nonlinear least squares problem $\min _{c^{(k+1)}}\left\|A^{(k+1)}-P^{(k)} S^{*} Q^{(k) t}\right\|_{F}^{2}$. This problem can be solved by equating the gradient of $\left\|A^{(k+1)}-P^{(k)} S^{*} Q^{(k) t}\right\|_{F}^{2}$ to zero and solving the linear system resulting $A_{t r} c^{(k+1)}=b_{t r}^{(k)}$, where [7] $A_{t r}=\left[\operatorname{tr}\left(A_{i}^{t} A_{r}\right)\right]_{r, i=1, l}$ and $b_{t r}=$ $\left[\operatorname{tr}\left(A_{r}^{t}\left(X^{(k)}-A_{0}^{t}\right)\right)\right]_{r=1, l}$. This LP algorithm converges to a stationary point in the sense that [7] $\left\|A^{(k+1)}-X^{(k+1)}\right\|_{F} \leq\left\|A^{(k)}-X^{(k)}\right\|_{F}$.

\subsection{MIII}

The method MIII described here is the method presented in [3] by Chu. This method finds the intersection of $\Gamma\left(S^{*}\right)$ and $\Lambda(c)$, defined in (2.3), using an iterative Newton-like method. In the iteration $k$, given $X^{(k)} \in \Gamma\left(S^{*}\right)$, there exist matrices $P^{(k)}$ and $Q^{(k)}$ such that $X^{(k)}=P^{(k)} S^{*} Q^{(k) t}$ and the tangent vector to $\Gamma\left(S^{*}\right)$ which starts from the point $X^{(k)}$ and crosses $A\left(c^{(k+1)}\right)$, can be expressed as

$$
X^{(k)}+X^{(k)} L^{(k)}-H^{(k)} X^{(k)}=A\left(c^{(k+1)}\right)
$$

where $L^{(k)} \in \Re^{n \times n}$ and $H^{(k)} \in \Re^{m \times m}$ are skew-symmetric matrices. Because $X^{(k)}=P^{(k)} S^{*} Q^{(k) t}$, (8) can be expressed as

$$
S^{*}+S^{*} \tilde{L}^{(k)}-\tilde{H}^{(k)} S^{*}=W^{(k)},
$$


where $\tilde{L}^{(k)}=Q^{(k) t} L^{(k)} Q^{(k)}, \tilde{H}^{(k)}=P^{(k) t} H^{(k)} P^{(k)}, W^{(k)}=P^{(k) t} A\left(c^{(k+1)}\right) Q^{(k)}$. Equating the diagonal elements of (9), we obtain the linear system (7), that calculate $c^{(k+1)}\left(A\left(c^{(k+1)}\right)\right.$ and $\left.W^{(k)}\right)$. Equating the off-diagonal elementos of (9), we calculate $\tilde{H}^{(k)}$ and $\tilde{L}^{(k)}$ [3].

In order to calculate $X^{(k+1)}$ from $A\left(c^{(k+1)}\right)$, e.g. $P^{(k+1)}$ and $Q^{(k+1)}$, matrix $A\left(c^{(k+1)}\right)$ must be lifted to a point in $\Gamma\left(S^{*}\right)$. Then $X^{(k+1)}$ is defined as $X^{(k+1)}=P^{(k+1)} S^{*} Q^{(k+1) t}$ and $P^{(k+1)}$ and $Q^{(k+1)}$ are orthogonal matrices which can be approximated by $P^{(k+1)} \approx P^{(k)} R$ and $Q^{(k+1)} \approx Q^{(k)} T$, being $R$ and $T$ the Cayley transforms: $R=\left(I+\frac{1}{2} H^{(k)}\right)\left(I-\frac{1}{2} H^{(k)}\right)^{-1}$ and $T=$ $\left(I+\frac{1}{2} L^{(k)}\right)\left(I-\frac{1}{2} L^{(k)}\right)^{-1}$. See [1] and [3] for details.

\section{$3 \quad$ Numerical Experiments}

In order to observe the behaviour of the algorithms when $m, n>5$, they are analyzed experimentally, evaluating memory requirements, convergence, accuracy and efficiency. By each analyzed aspect, we compare the algorithms to determine those most suitable to solve the IASVP.

The numerical experiments have been carried out taking matrices sizes of $m=n=\{5,10,15,20,25,30,50\}$, using random values for matrices and vectors of the IASVP and taking different initial guesses $c^{(0)}$.

The algorithms have been implemented in Matlab and executed in a $2.2 \mathrm{GHz}$ Intel Xeon biprocessor with 4 GBytes of RAM and operating system Linux Red Hat 8.

Memory Requirements. The storage required for $n+1$ matrices of size $m \times n$ $\left(A_{i}, i=1: n\right)$, two vectors of size $n\left(S^{*}, c\right)$ and the vectors and matrices required by each algorithm (singular values and vectors, Jacobians matrices, skew-symmetric matrices, ...), can be seen in Table 1. The FB method has the greatest memory needs, thereby is hardly feasible to implement for $m>50$. The best algorithms from this point of view are MI and LP.

Table 1. Memory Requirements in $\operatorname{KBytes}(\mathrm{KB})$, MBytes(MB), GBytes(GB) and TBytes(TB)

\begin{tabular}{l|rrrrrrr}
\hline$m=n$ & 4 & 10 & 30 & 50 & 100 & 500 & 1000 \\
\hline $\mathrm{BF}$ & $12 \mathrm{~KB}$ & $366 \mathrm{~KB}$ & $28 \mathrm{MB}$ & $205 \mathrm{MB}$ & $4 \mathrm{~GB}$ & $2 \mathrm{~TB}$ & $32 \mathrm{~TB}$ \\
$\mathrm{MI}$ & $1.2 \mathrm{~KB}$ & $12 \mathrm{~KB}$ & $250 \mathrm{~KB}$ & $1.1 \mathrm{MB}$ & $8.3 \mathrm{MB}$ & $1 \mathrm{~GB}$ & $8 \mathrm{~GB}$ \\
$\mathrm{LP}$ & $1.2 \mathrm{~KB}$ & $12 \mathrm{~KB}$ & $250 \mathrm{~KB}$ & $1.1 \mathrm{MB}$ & $8.3 \mathrm{MB}$ & $1 \mathrm{~GB}$ & $8 \mathrm{~GB}$ \\
$\mathrm{MIII}$ & $1.7 \mathrm{~KB}$ & $15 \mathrm{~KB}$ & $270 \mathrm{~KB}$ & $1.2 \mathrm{MB}$ & $8.6 \mathrm{MB}$ & $1 \mathrm{~GB}$ & $8.1 \mathrm{~GB}$ \\
\hline
\end{tabular}

Convergence. The convergence of the algorithms BF, MI and MIII is really sensitive to the initial guess. When the initial guess is taken as $c_{i}^{(0)}=c_{i}^{*}+\delta$ $(i=1: n)$, for small $\delta$ such as $\delta=0.1$ all of them converge; but when $\delta=1.0$ 


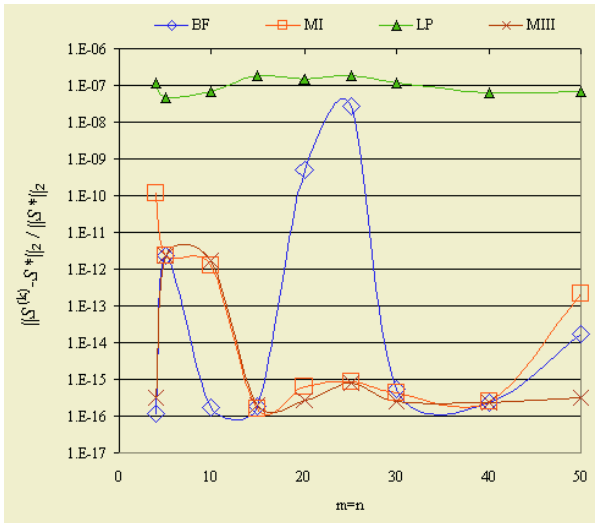

Fig. 1. Accuracy of Algorithms for different values of $m$, with $c_{i}^{(0)}=c_{i}^{*}+0.1(i=1: n)$

they only converge for sizes $m<50$ and when $\delta=10.0$ they only converge for $m<10$. Then, as, the initial guess is farther from the solution, these algorithms converge only for smaller problem sizes. This phenomenon is not suffered by LP, since it has the property $\left\|A^{(k+1)}-X^{(k+1)}\right\|_{F} \leq\left\|A^{(k)}-X^{(k)}\right\|_{F}$; 7 .

Solution Accuracy. The accuracy of the solution is measured by obtaining the relative errors of the obtained singular values with respect to the correct ones (Figure 4), with $\delta=0.1$. MIII gives the approximations $S^{*}$, while LP gives the worst; however, the LP errors are smaller than $1 e-6$.

Time Complexity estimates and Execution Times. The time complexity estimates are a function of the problem size $(m, n)$ and of the number of iterations to convergence $(K)$; this estimates can be split in two, the cost of the start-up phase $\left(T(m, n)_{\text {start } U p}\right)$ and the cost of each iteration inside the main

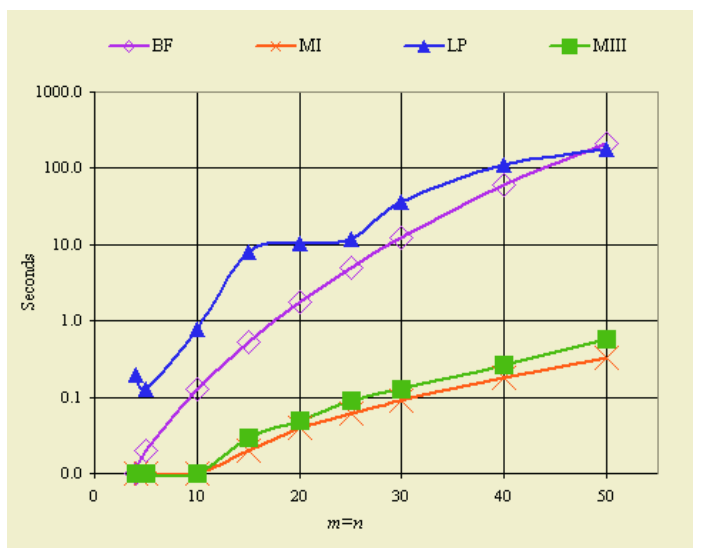

Fig. 2. Execution times (seconds) required to reach convergence, with different values of $m$ and $\delta=0.1$ 
Table 2. Number of iterations required for convergence with $\delta=0.1$

\begin{tabular}{l|ccccccccc}
\hline$m=n$ & 4 & 5 & 10 & 15 & 20 & 25 & 30 & 40 & 50 \\
\hline $\mathrm{BF}$ & 3 & 2 & 3 & 3 & 3 & 3 & 3 & 3 & 3 \\
$\mathrm{MI}$ & 2 & 2 & 2 & 3 & 3 & 3 & 3 & 3 & 3 \\
LP & 365 & 206 & 561 & 3398 & 2629 & 1998 & 4048 & 6304 & 5303 \\
MIII & 3 & 2 & 2 & 3 & 3 & 3 & 3 & 3 & 4 \\
\hline
\end{tabular}

loop $\left(T(m, n)_{\text {loop }}\right)$ so that the time complexity can be written as: $T(m, n, K)=$ $T(m, n)_{\text {start } U p}+K T(m, n)_{l o o p}$. Then, the estimates for each method (for $m=n$ ) can be written as:

$$
\begin{aligned}
& T(m, K)_{B F} \approx(62 / 3) m^{3}+O\left(m^{2}\right)+K\left\{(16 / 3) m^{6}+O\left(m^{5}\right)\right\} \\
& T(m, K)_{M I} \approx(44 / 3) m^{3}+O(m)+K\left\{2 m^{4}+\left(m^{3}\right)\right\} \\
& T(m, K)_{L P} \approx m^{4}+O\left(m^{3}\right)+K\left\{(56 / 3) m^{3}+O\left(m^{2}\right)\right\} \\
& T(m, K)_{M I I I} \approx(56 / 3) m^{3}+O\left(m^{2}\right)+K\left\{2 m^{4}+O\left(m^{3}\right)\right\}
\end{aligned}
$$

The time estimate of BF shows that it is not an acceptable algorithm. On the other hand, LP has the smallest time estimate in the iterative part, but it needs many more iterations for convergence (and execution time) than the other methods. This can be checked in Table 2 where the number of iterations $K$ is shown for some experiments, and in Figure 2, where the execution times are shown.

\section{Conclusions}

We have implemented and analyzed a set of algorithms for resolution of the IASVP and we have analized their behaviour for different values of $m(m>5)$.

The study carried out shows that the FB approach must be discarded as a practical algorithm given its high costs, both in memory $\left(O\left(m^{4}\right)\right)$ and execution time $\left(O\left(m^{6}\right)\right)$.

From the memory point of view, MI and LP are those of smaller requirements of memory; from the execution time point of view, MI is most efficient. The highest accuracy of the solutions, in most of the tested cases, is reached with MIII.

However, MI and MIII, like BF, have the drawback of being too sensitive to the quality of the initial guess. This sensitivity becomes worse as the size of the problem increases. In contrast, LP does not suffer this problem.

Since none of the algorithms possess properties good enough on its own, it seems desirable to combine several algorithms in a single one. A possible followup of this work is the development of this kind of algorithms; some approaches can be found in [2] and [7. 
The problem of the high costs of storage and execution times for problems of larger size $(m>50)$ can be tackled by parallelizing the algorithms, so that any processor involved stores only a part of the data structures and executes part of the calculations.

\section{Acknowledgement}

This work has been supported by Spanish MCYT and FEDER under Grant TIC2003-08238-C02-02 and DGIT-SUPERA-ANUIES (México).

\section{References}

1. Chan, R., Bai, Z., Morini, B.: On the Convergence Rate of a Newton-Like Method for Inverse Eigenvalue and Inverse Singular Value Problems. Int. J. Appl. Math., Vol. 13 (2003) 59-69

2. Chen, X., Chu, M.T.: On the Least Squares Solution of Inverse Eigenvalue Problems. SIAM, Journal on Numerical Analysis, Vol. 33, No. 6 (1996) 2417-2430

3. Chu, M.T.: Numerical Methods for Inverse Singular Value Problems. SIAM, Journal Numerical Analysis, Vol. 29, No. 3 (1992) 885-903

4. Chu, M.T.: Inverse Eigenvalue Problems. SIAM, Review, Vol. 40 (1998)

5. Bai, Z., Morini, B., Xu, S.: On the Local Convergence of an Iterative Approach for Inverse Singular Value Problems. Submitted

6. Flores G., Vidal A.: Paralelización del Método de Elevación y Proyección para la Resolución del Problema Inverso de Valores Singulares. Primer Congreso Internacional de Computación Paralela, Distribuida y Aplicaciones, (2003).

7. Flores, G., Vidal A.: Parallel Global and Local Convergent Algorithms for Solving the Inverse Additive Singular Value Problem. Submitted

8. Friedland, S., Nocedal, J., Overton, M.L.: The Formulation and Analysis of Numerical Methods for Inverse Eigenvalue Problems. SIAM, Journal on Numerical Analysis, Vol. 24, No. 3 (1987) 634-667

9. Groetsch, C.W.: Inverse Problems. Activities for Undergraduates. The mathematical association of America (1999)

10. Kelley, C.: Iterative Methods for Linear and Nonlinear Equations. SIAM (1995)

11. Neittaanmki, P., Rudnicki, M., Savini, A.: Inverse Problems and Optimal Design in Electricity and Magnetism. Oxford: Clarendon Press (1996)

12. Sun, N.: Inverse Problems in Groundwater Modeling. Kluwer Academic (1994) 